\title{
Post-operative parenteral nutrition
}

By A. W. Wilkinson, Institute of Child Health, 30 Guilford Street, London, WC

All injury, accidental or surgical, if sufficiently severe, has profound effects on the function of the alimentary tract as well as on other parts of the body. The majority of surgical patients are well able to stand for a period of $24-48 \mathrm{~h}$ deprivation of both water and food, provided they do not also suffer large extra-renal losses. For some years it has been the objective of many surgeons to avoid this post-operative starvation and to provide materials for the healing of injuries they have inflicted by supplying suitable materials to the patients by continuous intravenous infusion, and this is what is implied by 'parenteral nutrition after injury'. There is no doubt that this type of feeding has great value in a limited range of circumstances and that for some patients it is the means of prolonging if not preserving their life.

One of the difficulties in interpreting many previous surgical metabolic studies lies in the wide variation in the ambient temperature in which the patients were treated; many protocols do not mention ambient temperature, which may extend over a wide range in any country with a continental type of climate. It seems possible that, in a suitably elevated ambient temperature, what was formerly regarded as the total metabolic response to injury may be greatly modified or even abolished. There are many interesting possibilities about the response to injury at higher ambient temperatures which have a bearing on parenteral feeding. For example, can the large potassium loss in the urine during the first $24-48 \mathrm{~h}$ after injury at an ambient temperature of $21^{\circ}$ be reduced in the same way as the nitrogen loss is so much reduced by raising the temperature to $32^{\circ}$ ? Is this $\mathrm{K}$ loss related more to the intracellular dehydration associated with injury than to protein catabolism and is there any justification for the early parenteral replacement of this $\mathrm{K}$ loss?

Post-operative parenteral nutrition implies the provision of all the requirements of the injured body both for maintenance and daily requirements; for the neonate or young infant, the needs of growth must be provided also. It is now evident from the work of Caldwell (1962) and Cuthbertson and his co-workers (Campbell \& Cuthbertson, 1967; Cuthbertson, Smith \& Tilstone, 1968) that our former apparent certainty about the requirements after injury has largely vanished and that we must start again and make fresh observations on the human subjects of various ages. Until we do so estimation of the requirements of protein and various sources of nonprotein calories for the post-operative patient will remain partly guesswork.

When neonates and adults are fed totally by intravenous infusion their different daily needs must be applied to the design of the solutions and materials which are to be used. In some of the amino acid solutions now available the concentration of sodium may be $50 \mathrm{~m}$-equiv. $/ 1$, of $\mathrm{K}$ only $10 \mathrm{~m}$-equiv. $/ 1$ and of magnesium $5 \mathrm{~m}$-equiv. $/ 1$ or less. About $7 \mathrm{~d}$ after birth the neonate fed on human breast milk receives about $8 \mathrm{~m}$-equiv. $\mathrm{K}$, about $5 \mathrm{~m}$-equiv. $\mathrm{Na}$ and $1.7 \mathrm{~m}$-equiv. $\mathrm{Mg}$ in $24 \mathrm{~h}$. Expressed 
per $\mathrm{kg}$ body-weight the neonate thus requires $0.3 \mathrm{~g} \mathrm{~N}, 2.3 \mathrm{~m}$-equiv. $\mathrm{K}, \mathrm{I} \cdot 4 \mathrm{~m}$-equiv. $\mathrm{Na}$ and $0.5 \mathrm{~m}$-equiv. $\mathrm{Mg}$ and about $120 \mathrm{kcal}$, whereas the adult requires $0.2 \mathrm{~g} \mathrm{~N}$, $\mathrm{I} \cdot 2 \mathrm{~m}$-equiv. $\mathrm{K}, 2 \mathrm{~m}$-equiv. $\mathrm{Na}, 0.2 \mathrm{~m}$-equiv. $\mathrm{Mg}$ and $35 \mathrm{kcal} / \mathrm{kg}$ body-weight.

The renal functional capacity of the newly born is very different from that of the adult. This difference seems to be mainly related to the natural diet of the neonate, human breast milk. Of the amount of milk consumed, $80-85 \%$ is absorbed from the gut and about $65 \%$ is converted to tissue. Thus only about $x_{5}-20 \%$ of the intake is available for excretion in the urine. The human neonate has remarkable powers of conservation of substances such as $\mathrm{Na}$ and $\mathrm{K}$ but very poor powers of excretion of large, added excesses. Both vomiting and diarrhoea may protect the body by preventing the absorption of too much of an excessive oral intake; when food and fluids are administered by intravenous infusion these two safeguards are short-circuited. The disposal of water and other substances added direct to the circulation by infusion depends direct on the requirements and excretory capacities of the subject and in the neonate, in particular, the inability to excrete large added loads of water imposes the most important limitation on the scope of parenteral nutrition.

At the end of the ist week of life insensible loss of water through the skin amounts to about $90 \mathrm{ml} / 24 \mathrm{~h}$, the obligatory volume of urine is about $30 \mathrm{ml} / 24 \mathrm{~h}$ and any further urine volume is dependent on how much additional water is consumed. The normal intake of milk at the end of a week is about $400 \mathrm{ml}$. This volume of milk can be tolerated day after day mainly because only about $15 \%$ of the total intake of water in milk is available for excretion. Thus tolerance of a volume of $400 \mathrm{ml} / \mathrm{d}$ seems to depend on the simultaneous administration of materials which are suitable for conversion to protoplasm together with enough non-protein calories to safeguard the administered amino acids. The solution should also contain suitable minerals in the proper proportions and should be buffered to raise its $\mathrm{pH}$ of 5.6 to something nearer normal. The first preparations used for intravenous feeding were acid or enzymatic hydrolysates of casein or fibrin and contained a large amount of polypeptides. Later the peptides were removed by dialysis and now 3 or $5 \%$ solutions of synthetic amino acids are available. Non-protein calories can be provided as $5 \%$ solution of glucose, 3 or $5 \%$ ethanol or 5 or $10 \%$ fructose or sorbitol but the most compact source is a 10 or $20 \%$ emulsion of soya-bean oil.

It is a nutritional principle that protein should provide not more than $12.5 \%$ of the total kcal in the 24-h intake of food, no matter how this may be conveyed to the body. In arranging the combination of fluids for intravenous feeding it is probably simplest first to establish the $24-\mathrm{h}$ intake of $\mathrm{N}$ and then to calculate the calories which could be derived from it. The remaining $87.5 \%$ of the total calorie intake is then divided between available non-protein sources such as glucose, ethanol, sorbitol, fructose and fat.

Parenteral feeding may be used for short or long periods. For example, almost 20 years ago Stare and his colleagues (Van Itallie, Waddell, Geyer \& Stare, 1952) kept a severely injured man alive for $67 \mathrm{~d}$ solely by the intravenous administration of a fat emulsion, glucose and protein hydrolysate. Abbott, Krieger, Holden, Bradshaw \& Levey (1957) reported that they had been able to abolish starvation during 
the Ist week after partial gastrectomy by the administration of fat emulsions with amino acids, glucose and alcohol. More recently Wilmore, Groff, Bishop \& Dudrick (1969) using a $5 \%$ fibrin hydrolysate and a $22 \%$ glucose were able to maintain for $400 \mathrm{~d}$ from birth a baby from whom all but $10 \mathrm{~cm}$ of small intestine had been resected.

The longer parenteral feeding continues the more complicated it becomes. Trace elements must be provided. Wilmore et al. (1969) achieved this by giving human plasma once a week, and some of the most recently developed preparations include trace elements. Vitamins also must be supplied by injection if parenteral feeding lasts for more than ro $\mathrm{d}$. With some of the earlier fat emulsions there was a risk of spontaneous bleeding if the infusion went on too long but there seems to be little risk of this with the soya-bean emulsions which are now available.

The indications for the use of parenteral feeding must be considered individually for each patient. The capacity of a well-nourished human subject to survive for $4 \mathrm{~d}$ or more in good condition without any intake of fluid or food should always be remembered before decisions are made to start intravenous feeding. Ultimately the patient must be able to survive on what can be taken by mouth and digested in the gut. Intravenous feeding can be used only as long as there are accessible unthrombosed veins and it may lead to severe and sometimes fatal complications. When enough of the alimentary tract, especially the distal small intestine, remains it is simpler and safer to feed through a gastrostomy or jejunostomy than by intravenous infusion. In head injuries, for example, intravenous feeding is seldom indicated provided a tube can be passed into the stomach or inserted into the upper small, intestine. Recently, parenteral feeding has been used in renal failure but in the end oral or tube feeding may turn out to be safer as well as a simpler procedure than continuous feeding by vein. In patients with low fistulas of the small intestine, intravenous feeding probably has considerably more to offer than any other type of nutrition, especially if all oral intake is stopped and the nutritional state of the patient can be so improved that the fistula is able to heal. The same is often true in ulcerative colitis and Crohn's disease and some other malabsorptive states such as disaccharide intolerance which in the neonate or infant is now the commonest indication for parenteral feeding. It may also be necessary in severe enterocolitis secondary to Hirschsprung's Disease, or for short infusions of 4-5 $\mathrm{d}$ in babies who have already suffered from so much starvation and depletion of their body fluids that it would be impossible for them to survive if an additional 5 -d period of starvation was imposed. It is also of value in babies in whom so much intestine has been resected or destroyed by infarction by volvulus or gangrene in an intersusception that too little remains for the large digestive and absorptive requirements of the neonate or small infant.

There is still discussion about whether amino acids and carbohydrate or ethanol solution should be always administered at the same time as fat emulsion. When an intravenous infusion of amino acids and accompanying calories is started there is usually an increase in the $\mathrm{N}$ output in the urine. While this may be due to the inadequate provision of non-protein calories or because these calories were not given 
in sufficient quantities simultaneously with the amino acids it must also be remembered that the plane of protein metabolism has been reset at a much higher level than before. The proportions of the individual amino acids in a solution may be in accord with the recommendations of Rose ( 1949), but be less well utilized when administered all together into the venous blood stream. Perhaps the different rates at which amino acids, fat and carbohydrate reach the blood stream and the liver after the oral consumption of a meal is more important than has been thought.

The most important limiting factor in the use of intravenous feeding is the chemical thrombophlebitis which is such a common sequel of the use of a peripheral vein for more than a few hours. This is probably due to the $\mathrm{pH}$ of the combined solution of amino acids and carbohydrate usually being as low as $5^{\circ} 0$ or $5^{.6}$. This thrombophlebitis can be to some extent avoided by changing the vein every $24 \mathrm{~h}$ or even more frequently if redness and tenderness are found. When there is infection as well as thrombophlebitis septicaemia may ensue and carries a high mortality rate. To avoid this it has been recommended that the irritant solutions should be more rapidly diluted by being administered into the larger stream of blood in the inferior vena cava, reached through the long saphenous vein in the thigh, or the superior vena cava reached through the subclavian vein. Even so, thrombosis of the superior or inferior vena cava has occurred. We have maintained, like others have done, a child for $35 \mathrm{~d}$ entirely by infusions through superficial veins mostly on the trunk after those in the limbs became thrombosed.

When there is a real need for parenteral nutrition there is likely also to have been large extra-renal losses of fluid from the body by vomiting, diarrhoea or discharge from a fistula. For such a patient it is essential that the extra-renal losses should be collected and that their content at least of $\mathrm{Na}, \mathrm{K}$ and $\mathrm{Mg}$ should be measured. It is then possible to calculate much more specifically replacements of the extra-renal losses as well as the daily requirements for nutrition and repair or growth. Particularly in the very young baby, the disturbances in chemical equilibrium which result from large extra-renal losses, particularly of gastro-intestinal secretions, make it necessary to follow the $\mathrm{pH}$ and base excess of the extracellular fluid with great care especially as most of the preparations used for intravenous feeding are acid in reaction. Blood should be withdrawn and centrifuged at least every $24 \mathrm{~h}, 4 \mathrm{~h}$ after the last fat was administered to make sure that fat is being cleared satisfactorily from the plasma. Modern soya-bean oil emulsions are well tolerated and metabolized even by the seriously-ill, newly born baby.

When food cannot be taken by mouth or mixtures of milk and other foodstuffs cannot be introduced by gastrostomy or enterostomy, parenteral feeding may be of much value. Survival may depend on it and normal growth of even the newly born can apparently be maintained (Wilmore et al. 1969). Success in this technique depends the appreciation and careful observance of nutritional principles and on the proper use of the materials which are available. Failure to do this, in particular to provide too few calories and inadequate cover of the protein with non-protein calories, is common in clinical practice and results in the waste of both the opportunity and the materials.

$30(2) 6$ 
Abbott, W. E., Kreiger, H., Holden, W. J., Bradshaw, J. \& Levy, S. (1957). Metabolism 6, 69r. Caldwell, F. T. (1962). Ann. Surg. 155, i19.

Campbell, R. M. \& Cuthbertson, D. P. (I967). Q. Fl exp. Physiol. 52, i14.

Cuthbertson, D. P., Smith, C. M. \& Tilstone, W. J. (ro68). Br. F. Surg. 55, 513.

Rose, W. C. (1949). Fedn Proc. Fedn Am. Socs exp. Biol. 8, 546.

Van Itallie, T. B., Waddell, W. R., Geyer, R. P. \& Stare, F. J. (r952). Archs intern. Med. 89, 353.

Wilmore, D. W., Groff, D. B., Bishop, H. C. \& Dudrick, S. J. (1969). F. Paediat. Surg. 4, $18 \mathrm{r}$.

\section{Feeding the unconscious patient}

\section{By Sally Day and Monamy Buckell*, Atkinson Morley's Hospital, Neurosurgical Unit, St George's Hospital, London, $S W_{\mathrm{I}}$}

This communication deals with the feeding of unconscious patients from the point of view of the hospital dietitian, and describes the choice, organization and assessment of tube feeds. The observations are the result of close collaboration between a dietitian and a chemical pathologist working with the neurosurgical unit of Atkinson Morley's Hospital where tube feeding is an important part of the total care of the unconscious patient.

There are forty-six adult and nine paediatric beds, and a quick turnover of cases with a high proportion of acutely-ill, comatose patients. In this situation the dietitian is called upon to provide feeds that will cover the patient's maintenance requirements and repair the deficits, also taking into account the problems peculiar to cerebral damage and the unconscious state. These patients are even more dependent than babies, they cannot cry when they are starving or thirsty or have a headache, and, in addition, their homoeostatic mechanisms may have broken down so that the fluid and electrolyte content of their feeds becomes a critical matter.

The advantages of nasogastric feeding are listed in Table $\mathrm{I}$. In terms of total calories, and of carbohydrate, protein and fat we can give a complete diet and supplements of iron and vitamins can be easily added as appropriate. When absorption from the gut is satisfactory, fluid balance can usually be controlled via the feeds. However, when fluid and electrolyte balance is critical, intravenous therapy may be needed in addition.

\section{Table 1. Advantages of nasogastric tube feeding}

1. Nutritional requirements covered

2. Drugs, electrolytes and nutritional supplements given via nasogastric tube

3. Generally well tolerated and easily modified

4. Ease of preparation, storage and administration

5. Very low cost in comparison with intravenous preparations available

*Present address: The Maudsley Hospital, Denmark Hill, London SE5. 